\title{
USANDO ESTRATÉGIAS COGNITIVAS DE ESTUDANTES TALENTOSOS COMO APOIO PARA O DESENVOLVIMENTO DA CAPACIDADE DE RESOLUÇÃO DE PROBLEMAS EM MATEMÁTICA.
}

\author{
Eliane Vigneron Barreto Aguiar, Instituto Federal Fluminense RJ, \\ elianevig@yahoo.com.br \\ Liane Margarida Rockenbach Tarouco, Universidade Federal do Rio Grande do Sul, \\ liane@penta.ufrgs.br \\ Eliseo Berni Reategui, Universidade Federal do Rio Grande do Sul, \\ eliseoreategui@gmail.com
}

\begin{abstract}
RESUMO. Este artigo apresenta o estágio atual de uma investigação que busca capturar estratégias e processos cognitivos utilizados por alunos talentosos durante a resolução de problemas de Matemática com vistas a disponibilizá-las para os demais estudantes mediante o uso de um sistema de raciocínio baseado em casos. Este sistema opera como um assessor mostrando estratégias cabíveis (já usadas por estudantes talentosos) e que podem ser experimentadas pelos demais estudantes no processo de resolução de novos problemas contribuindo para desenvolver as habilidades cognitivas num processo de aprendizagem auto-regulatória.
\end{abstract}

PALAVRAS-CHAVE: processos cognitivos; estudantes talentosos; problemas; tecnologia.

\section{USING COGNITIVE STRATEGIES TO SUPPORT TALENTED STUDENTS TO DEVELOP PROBLEM-SOLVING ABILITY IN MATHEMATICS.}

\begin{abstract}
This paper presents an investigation on the capture of cognitive processes and strategies used by talented students during mathematics problem solving, as a way to make these strategies available to other students through a case-based reasoning system. This system works as an assistant showing possible strategies (already used by talented students) and that may be experimented by the other students in solving new problems, contributing to the development of cognitive skills in a self-adjusting learning process.
\end{abstract}

KEYWORDS: cognitive processes; talented students; problems; technology.

\section{INTRODUÇÃO}

Aprender a resolver problemas é a mais importante habilidade que os estudantes podem adquirir em algum momento da vida escolar, segundo afirma Jonassen (2003). Os novatos, na tentativa de resolver um problema, devem inicialmente observar e imitar o que fazem as outras pessoas mais experientes quando resolvem os seus problemas e, por fim, aprenderão a resolver problemas, resolvendo-os. Sendo "a resolução de problemas é uma habilitação prática” Polya (2006), ela precisa ser exercitada mas deve contar com o apoio e a orientação de uma pessoa ou entidade mais experiente.

Inerente ao processo de resolução de problema, existem desafios que se configuram como aspectos estimulantes e motivadores ao resolver um problema: a 
organização dos dados, determinação da estratégia, experimentação de um caminho, se não for o caminho adequado, voltar atrás e procurar outro, encontrar uma solução, verificar a validade da solução, analisar o processo de resolução, fazer uma generalização para novos dados ou novas situações, são etapas que deverão ser desenvolvidas e são fundamentais para o crescimento individual, (Loureiro et al., 1990). Estudantes talentosos suplantam estes desafios com maior facilidade. Estudar as estratégias e processos cognitivos dos estudantes talentosos pode contribuir para auxiliar a delinear estratégias de ensino-aprendizagem que possam alavancar o processo de resolução de problemas pelos demais estudantes, que estão sendo designados neste estudo como estudantes de inteligência normal.

(Gardner, 1995) define talento "por um arranjo complexo de aptidões ou inteligências, habilidades instruídas e conhecimento, disposições de atitudes de motivações que predispõem um indivíduo a sucessos em uma ocupação, vocação, profissão, arte, ou negócio”. Alunos que resolvem com eficácia problemas de Matemática, possuem um talento especial para isto e são chamados de alunos talentosos. Os alunos talentosos que participam deste estudo são alunos medalhistas da OBMEP - Olimpíada Brasileira de Matemática das Escolas Públicas.

Existem evidências científicas de que é desde cedo que os estímulos devem ser fornecidos aos estudantes com talento especial para a Matemática, porque "em nenhuma outra etapa da vida eles estão tão propensos a ser criativos com os números”. Martin Grötschel, especialista alemão da Universidade Técnica de Berlim, afirma que "estudos mostram que, até cerca dos 20 anos, os jovens ainda não mecanizaram os caminhos para solucionar os problemas, o que deixa o cérebro mais livre para o exercício da criatividade - fundamental para avançar nesse campo”. Pode-se citar alguns exemplos que comprovam esta teoria e que ajudam a entender por que tantos gênios da matemática surgiram ainda na adolescência: o francês Blaise Pascal (1623 - 1662), com apenas 16 anos, criou seus primeiros teoremas na área da geometria e, o americano John Nash, aos 21 anos, escreveu sua tese sobre a teoria dos jogos, que lhe rendeu o Prêmio Nobel de Economia em 1994 (Revista Veja, maio 2010, ed.2165).

De acordo com técnicos do MEC - Ministério da Educação (Brasil, 2008), pessoas com altas habilidades/superdotação apresentam elevada criatividade, grande envolvimento na aprendizagem e na realização de tarefas em áreas de seu interesse e, além disso, demonstram elevado potencial (isolado ou combinado) intelectual, acadêmico, em liderança, psicomotricidade e artes. Segundo (Fernandes e Viana, 2009), "a incidência de altas habilidades/superdotação ocorre de modo aleatório, numa estimativa de 3 a $5 \%$ da população mundial, tanto em grupos privilegiados ou marginalizados socialmente”.

“O pensamento dos aprendizes talentosos é dito idiossincrático, ou seja, é uma característica comportamental ou estrutural peculiar aos alunos talentosos” (Rogers, 2007). Isto significa que um único aprendiz talentoso, não necessariamente, exigirá uma reconceitualização de estudos já realizados, em pesquisas, a partir de como apropriadamente e completamente funciona o pensar deste aprendiz. Em seu estudo Rogers apresenta opções de trabalhar a aprendizagem dos aprendizes talentosos de forma a aumentar suas habilidades, uma vez que estes estudantes precisam de algumas oportunidades para trabalhar independentemente e para desenvolver completamente os talentos demonstrados.

Codificação, comparação e combinação são os três maiores componentes cognitivos, utilizados pelos alunos talentosos, responsáveis pelas soluções corretas dos problemas que envolvem discernimento. Dois componentes adicionais também têm sido freqüentemente considerados como inerentes ao processo de resolução de problemas: 
recuperação e direcionamento de objetivos, conforme (Sternberg e Davidson, 1983; Gorodetsky e Klavir, 2003).

Segundo (Jonassen, 2003) "existem pelo menos dois atributos críticos na definição de um problema. Primeiro, um problema é uma entidade desconhecida em algum contexto. Segundo, encontrar a solução ou resolver o problema desconhecido deve ter algum valor social, cultural ou intelectual”. Existem diferenças na abordagem dos estudantes talentosos e os demais ao se envolverem em resolução de problemas. Essas diferenças envolvem recuperar substancial quantidade de informações. Ao resolver um problema histórico, por exemplo, é necessário que o estudante faça a identificação de palavras chaves na história, seleção de algoritmo apropriado e seqüência de aplicação do algoritmo, reconhecimento da estrutura semântica do problema e que tenha a capacidade para seqüenciar suas corretas etapas de solução.

Ao analisar a resolução de problemas (troubleshooting), verifica-se que esses tipos de problemas envolvem examinar sistemas, organizar testes, avaliar resultados, formular hipóteses e confirmar estados de falhas e inspecionar situações prévias. Além disso, deve-se também tomar conhecimento dos testes realizados e seus resultados, que é bastante valioso para aprendizagem e que poderia ser diretamente apoiado pelo uso de um sistema de Raciocínio Baseado em Caso ${ }^{1}$. "Em atividades de troubleshooting, o uso de ações de diagnóstico é indispensável nos vários estágios do problema, e a integração destes resultados no sistema contribui para aumentar dinamicamente a avaliação da situação” (Melchiors, 1999).

Os problemas lógicos envolvem uma atividade de aprendizagem de controle lógico e manipulação de limitadas variáveis, dentro de um contexto abstrato, numa estrutura de descoberta, onde o critério de sucesso está numa eficiente manipulação das variáveis. Surge, portanto, a seguinte pergunta: o que diferencia os estudantes talentosos dos demais neste tipo de problema? Como um sistema apoiado em TIC pode ajudar os estudantes de inteligência normal a alcançarem o desempenho dos estudantes talentosos?

\section{UMA TECNOLOGIA COMO SUPORTE PARA APRENDIZAGEM}

Buscando uma tecnologia para o armazenamento dos processos cognitivos dos talentosos, para posterior recuperação e reuso pelos demais estudantes (estudantes de inteligência normal), e possibilitando que novos problemas sejam resolvidos, esta pesquisa propõe a criação e utilização de um Chatterbot com uma ampla base de conhecimento. O Chatterbot representa um grande potencial para a aprendizagem de resolver problemas dos estudantes de inteligência normal, uma vez que incorporam novas idéias, novos conceitos utilizando linguagem natural. Ele pode servir como um interpretador de dados, além de uma fonte de consulta durante o processo de resolver problemas em matemática tentando replicar uma solução apresentada por um estudante talentoso que é mais capacitado. "O objetivo principal de um sistema de chatterbot é o de simular uma conversação em linguagem natural para tornar esta comunicação o mais próxima possível da conversação de um ser humano” (Leonhardt, 2005).

A estratégia de conversação do Chatterbot é a estratégia utilizada para a escolha algorítmica da resposta mais adequada a cada interação com o usuário. A estratégia utilizada é a de Raciocínio Baseado em Caso que é uma técnica da Inteligência Artificial que utiliza o mecanismo lógico do raciocínio humano. O Raciocínio Baseado em Caso é aplicado a aprendizagem na resolução de problemas baseado em experiências

\footnotetext{
${ }^{1}$ Ou Case Based Reasoning - CBR que é uma técnica da Inteligência Artificial de resolver problema
} 
passadas e utiliza como método o armazenamento de casos pregressos. Segundo (Kolodner, 1993), “a efetividade do processo de raciocínio humano depende das experiências vividas, da associação adequada das experiências passadas com as novas situações, da adequação das adaptações nas soluções adotadas, da proficiência das avaliações e da habilidade de integrar novas experiências na memória para uso futuro”.

Este estudo prevê que este sistema funcione como um ambiente catalisador, para a partir das estratégias de resolver problemas dos talentosos, aprimorar nos estudantes de inteligência normal as habilidades cognitivas de codificação, combinação, comparação, objetivos direcionados e principalmente, a recuperação. Este processo de interação com pares mais capacitados, amplia a Zona de Desenvolvimento Proximal (Vygotsky, 2007) dos estudantes de inteligência normal, em função da intervenção dos estudantes que têm maior habilidade para resolver problemas, mesmo que esta intervenção não seja direta, mas intermediada por um sistema que armazene e permita recuperação seletiva de estratégias cognitivas.

Muitos sistemas baseados no computador têm sido propostos para aumentar o desempenho das pessoas quando aprendem individualmente, uma vez que "com a ajuda de modernas tecnologias, estudantes podem aprender eficientemente e atingir extraordinário desempenho” (Shih et al., 2010). Quando não recebem assistência, estudantes são menos efetivos em regular sua aprendizagem em seu ambiente de hipermídia. Assim, o uso de ferramentas de aprendizagem auto-regulada assistida pelo computador é relevante durante o processo de resolver problemas. Como exemplo, pode-se citar os sistemas de Troubleticket (Melchiors, 1999) que foram utilizados para armazenar problemas em rede de computador e atuavam como uma memória histórica da rede. Esta memória foi consolidada por um sistema especialista chamado DUMBO que incorporou um paradigma do raciocínio baseado em caso em um tradicional sistema de Troubleticket com o objetivo de usar o conhecimento armazenado nestes tickets para propor soluções para novos problemas.

"Desenvolver e implementar confiáveis propostas de pesquisa e ferramentas de pesquisa, bem como, estudar o impacto das questões organizacionais sobre a relação entre ensino de matemática e aprendizagem de matemática” (Tirosh, 2009) são os dois principais desafios para os próximos cinco anos, em seu domínio. Portanto, para o aprimoramento das habilidades cognitivas de resolver problemas nos estudantes de inteligência normal, assim como, desenvolvimento da capacidade de pesquisar, pensar criticamente e reflexivamente, usar e transformar a informação e tomar decisões propõese um sistema de aprendizagem auto-regulada com suporte de andaimes como apresentado a seguir.

\section{PROCESSOS DE APRENDIZAGEM}

O processamento da informação ocorrido na memória de um estudante talentoso fornece o entendimento de como estes estudantes utilizam sua memória, por exemplo, para resolver problemas de matemática. Visando aumentar a capacidade dos estudantes de inteligência normal de obtenção de sucesso na resolução de problemas matemáticos e buscando fazê-los utilizar processos cognitivos semelhantes aos talentosos, é proposta uma aprendizagem auto-regulada, com suporte de andaimes (subsunçores), que emprega metacognição e heurísticas, apoiada pela tecnologia, com o objetivo de desenvolver habilidades de aprendizagem independentes nesses estudantes e, conseqüentemente, a aquisição de uma aprendizagem significativa. 
A aprendizagem auto-regulada pode ser descrita como um processo de aprendizagem com quatro atributos (Schunk \& Zimmerman, 1994, apud Shih et al., 2010):

- Auto-motivação: Aprendizes auto-regulados possuem a tendência de manter um comportamento de aprendizagem com uma motivação muito forte. Aprendizes podem elevar esta motivação através de algumas práticas, tal como estabelecendo objetivos de aprendizagem.

- Planificação ou Automatização: Aprendizes auto-regulados são capazes de usar algumas estratégias junto com seus processos de aprendizagem, incluindo a utilização de estratégias auto-reguladas e cognitivas. Geralmente, aprendizes aumentam seus processos de aprendizagem quando usam estratégias autoreguladas em vez de estratégias cognitivas. Estratégias auto-reguladas contêm estabelecimento de objetivos, planejamento de objetivos, organização, transição, exercício, etc. Um aprendiz auto-regulado necessita efetivamente usar estratégias auto-reguladas para sua aprendizagem.

- Auto-consciência dos resultados do desempenho: Durante todo o processo de aprendizagem, aprendizes auto-regulados afiam suas auto-conscientizações através do seu comportamento de aprendizagem. Para aproximar-se de um resultado ideal, aprendizes auto-regulados devem estar conscientes de sua própria qualidade de aprendizagem e mudar o comportamento ou estratégia correspondente.

- Habilidade e sensibilidade ambiental/social: O ambiente e recursos de aprendizagem podem afetar o padrão de aprendizagem dos aprendizes. Aprendizes auto-regulados têm melhor habilidade em procurar recursos ou suporte de aprendizagem. Com tal habilidade, eles procuram combinar as condições ambientais e buscar outros recursos efetivamente.

A figura 3.1, apresenta um modelo cíclico da aprendizagem auto-regulatória (Zimmerman et al., 1996, apud Shih et al., 2010) que mostra como os aprendizes podem conseguir incorporar atributos da aprendizagem auto-regulada num sistema apropriado. Uma vez que, tais atributos sejam apropriados, os estudantes tem condições e habilidade para passar a auto-regular sua aprendizagem.

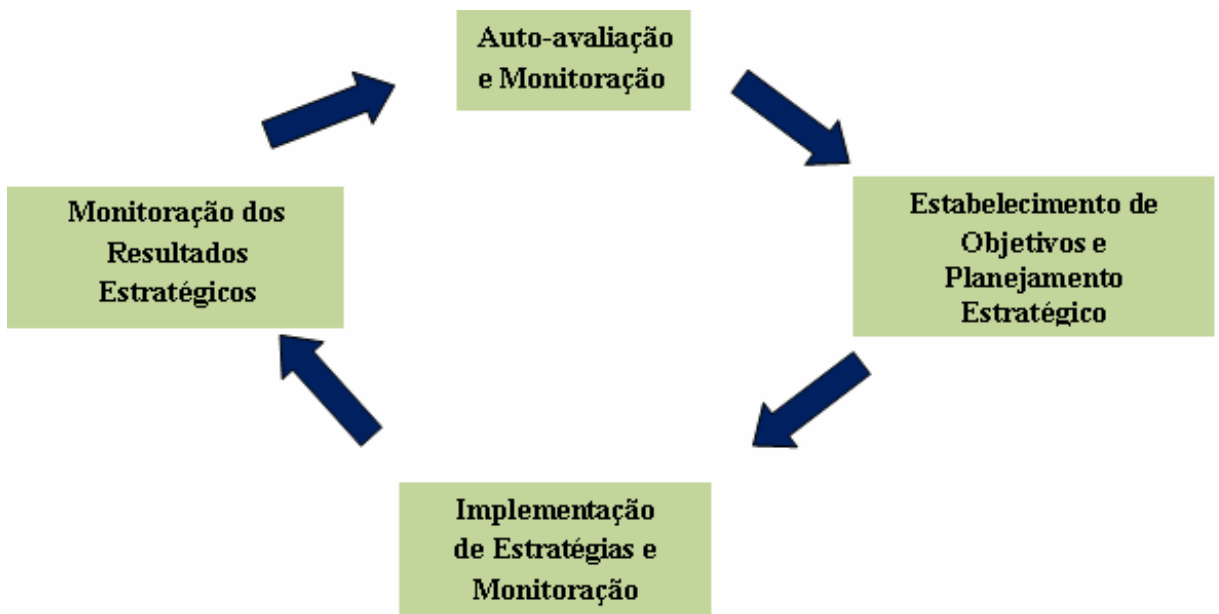

Figura 3.1 Modelo Cíclico de Aprendizagem Auto-regulatória (Zimmerman et al., 1996, apud Shih et al., 2010)

O ciclo, da figura 3.1, envolve quatro processos inter-relacionados que ajudam os aprendizes a avaliar seu desempenho e neste sentido capacita estudantes para organizar sua própria aprendizagem. Diversas alternativas em termos de uso da TIC 
(Tecnologia de Informação e Comunicação) têm sido consideradas como suporte aos processos de ensino e aprendizagem e em particular a este ciclo.

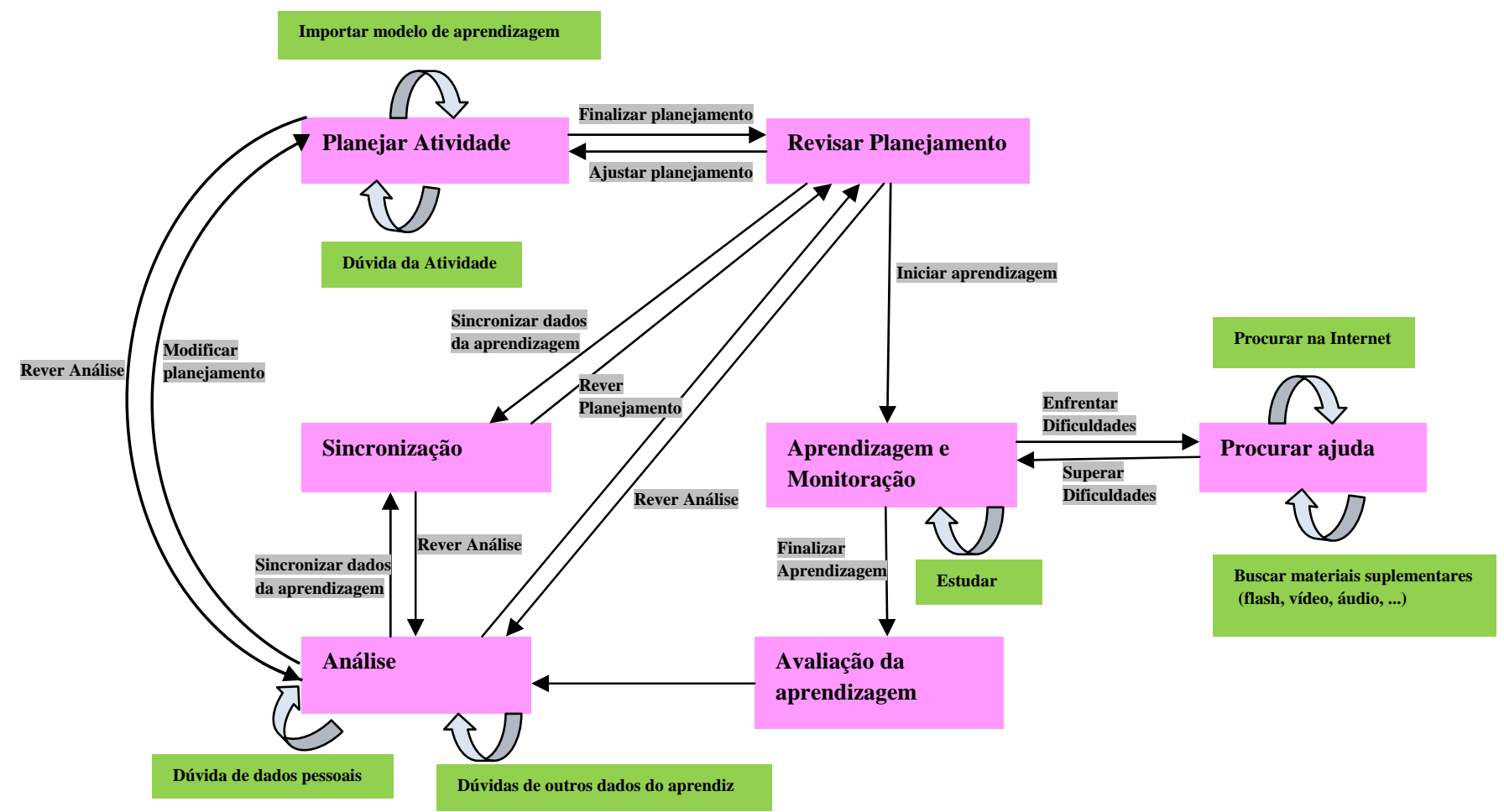

Figura 3.2 Diagrama de Transição do Estado do Sistema (Shih et al., 2005)

(Shih et al., 2005) propôs o protótipo de um sistema com ferramentas que podem suportar os atributos auto-regulatórios com apoio da teoria de andaimes, podendo ser utilizada para aprendizagem com auxílio da tecnologia (e-learning), e para aprendizagem móvel (m-learning). Este sistema proposto aumenta eficientemente, o desempenho dos aprendizes na aprendizagem auto-regulada, conforme feedback dos aprendizes e opiniões de teóricos educacionais. A figura 3.2 apresenta um diagrama de transição do estado que indica o comportamento de um aprendiz ao usar o modelo cíclico de aprendizagem auto-regulada para tornar-se auto-regulatório, conforme (Shih et al., 2005). O diagrama consiste de sete estados, indicando as ações de um aprendiz na aprendizagem auto-regulada. Entre estes sete, Planejar Atividade, Aprendizagem e Monitoração, Avaliação da Aprendizagem e Análise são os maiores estados que mapeiam os quatro processos no modelo cíclico de (Zimmerman et al., 1996, apud Shih et al., 2010) como apresentado na figura 3.1.

(Dabbagh \& Kitsantas, 2004, apud Shih, 2010) classificou as ferramentas pedagógicas baseadas na web (Web-based pedagogical tools - WBPT) em quatro classes: (1) ferramentas de comunicação e colaborativas; (2) ferramentas de ajuda e de criação de conteúdo; (3) ferramentas administrativas; e (4) ferramentas de avaliação. Estas ferramentas podem ser utilizadas como suporte dos atributos auto-regulatórios. Uma integração destas ferramentas deve ser estimulada para gradualmente apoiar os estudantes a adquirirem maior habilidade em aprendizagem auto-regulada. 


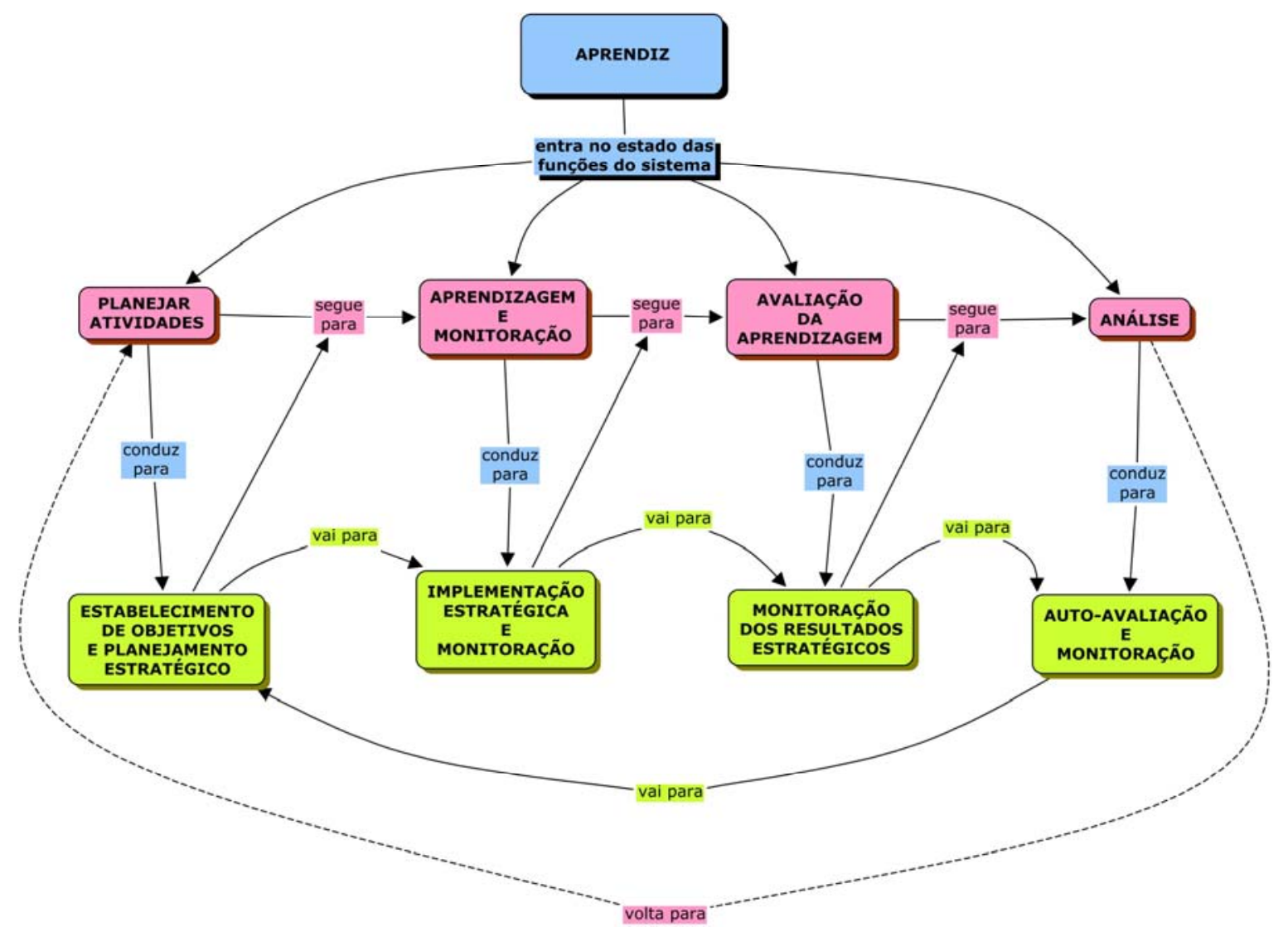

Figura 3.3 Funções do Sistema ligadas ao Modelo Cíclico Auto-regulatório.

A ligação existente entre os estados dos aprendizes no sistema: Planejar Atividade, Aprendizagem e Monitoração, Avaliação da Aprendizagem e Análise, e os quatro processos do modelo cíclico auto-regulatório, que são Estabelecimento de Objetivos e Planejamento Estratégico, Implementação Estratégica e Monitoração, Monitoração dos Resultados Estratégicos e, Auto-avaliação e Monitoração podem ser visualizados no mapa conceitual da figura 3.3. Segundo (Shih et al., 2010), através do sistema proposto, aprendizes no estado de Planejar Atividade podem obter informação sobre o que aprender e podem organizar devidamente o tempo necessário para aprendizagem, segundo a informação fornecida, isto conduz aos processos de Estabelecimento de Objetivos e Planejamento de Estratégias. Após estabelecer seus objetivos, aprendizes entram no estado de Aprendizagem e Monitoração. Como aprendizes podem comprometer-se em planejar atividades usando várias estratégias enquanto eles estão sendo observados, entra no estado do processo de Implementação Estratégica e Monitoração. No estado de Avaliação da Aprendizagem, aprendizes podem avaliar seus progressos através de testes. De acordo, com o mapa da figura 3.3, o aprendiz segue para o processo de Monitoração dos Resultados Estratégicos.

O mais importante processo de controle de desempenho que distingui aprendizes auto-regulados iniciantes de habilidosos é a auto-monitoração (Zimmerman \& Paulsen,1995 apud Shih et al., 2010). "Se aprendizes podem controlar seus próprios progressos, seus desempenhos acadêmicos, realizações, tempo sobre questões, comportamento em sala de aula, então habilidades de resolver problema podem ser aperfeiçoadas” (Lan, 1998, apud Shih et al., 2010). 
O processo de aprendizagem auto-regulada é um processo de metacognição, porque o aprendiz necessita controlar sua própria aprendizagem usando estratégias próprias para aperfeiçoar seu conhecimento. Aprendizes precisam tornarem-se habilidosos na aprendizagem auto-regulada com atividades de resolução de problemas de matemática e assim, precisam utilizar a metacognição para aumentar seus atributos auto-regulatórios. Etimologicamente, a palavra metacognição significa para além da cognição, isto é, a faculdade de conhecer o próprio ato de conhecer, ou, por outras palavras, consciencializar, analisar e avaliar como se conhece.

Através da metacognição, o sujeito-resolvedor de problemas matemáticos, tem informações sobre seu próprio processo de resolução, podendo supervisionar o resultado encontrado. Para compreender esse mecanismo utilizado pelos resolvedores, é preciso, em primeiro lugar, observar e acompanhar suas tendências cognitivas, de maneira a reconhecer seus próprios julgamentos e os elementos sobre os quais ele se apóia para justificar sua metacognição.

Tanto a metacognição, que é um processo utilizado durante a aprendizagem auto regulada, as heurísticas também são necessárias durante a aprendizagem auto-regulada. Ao utilizar o método heurístico, a atividade conduzida pelo professor deverá permitir que o aluno consiga, quando possível, descobrir sozinho as verdades matemáticas, para que o mesmo não torne-se um receptor passivo de conhecimentos. "Essa descoberta deverá se dar por intermédio da resolução de problemas, que visam orientar a pesquisa de teoremas e o desenvolvimento do raciocínio lógico, que serão orientados através de questionários intimamente coordenados”. Primeiramente, parte-se do conhecimento intuitivo, com exemplos concretos, para atingir posteriormente uma Matemática mais formal e dedutiva, de acordo com (Alvarez e Pires, 2000).

Um modelo heurístico na resolução de problemas de matemática pode ser encontrado em (Polya, 2006), que propõe os seguintes passos:

- Compreender o problema (conhecimento da incógnita, conhecimento dos dados, conhecimento das condições impostas) - essas heurísticas permitem que o(a) estudante certifique-se que considerou os aspectos relevantes do problema;

- Traçar um gráfico, fazer um diagrama, introduzir uma notação adequada - o sujeito tenta ver o problema por meio de notação simbólica, estabelecendo relações entre os elementos do problema;

- Estabelecer um plano (recordar um problema conhecido de estrutura idêntica) essa heurística supõe que o(a) estudante possua capacidade para fazer semelhanças, utilizar o pensamento analógico o qual permitirá chegar à solução do problema atual fazendo analogia com o já conhecido;

- Execução do plano (verificar passo a passo) - essa heurística permite dar segurança acerca da elaboração correta do plano de resolução do problema;

- Avaliação do plano (resolver o problema de maneira diferente) - essa heurística permite comprovar a solução obtida.

O método heurístico apóia-se no método indutivo, que tem como ponto de partida a intuição do aluno. Quando se trabalha com a intuição, faz-se uso de uma Matemática como ciência viva. É necessário que o aprendiz experimente, use a intuição e conseqüentemente, o pensamento indutivo até que compreenda os conceitos, para a partir de então, ser exigido dele o rigor (Alvarez e Pires, 2000).

O estudante na aprendizagem auto-regulada utiliza metacognição e o raciocínio heurístico para fazer com que novos conceitos sejam incorporados a um conceito já existente na estrutura cognitiva do aluno (subsunçor), alcançando uma aprendizagem significativa. A aprendizagem significativa (Ausubel, 1978) envolve a integração e transformação dos esquemas mentais existentes tendo em vista a nova informação. Se o 
estudante regular não tem esquemas mentais suficientemente amplos e complexos para acomodar a nova informação, ocorre aprendizagem mecânica, segundo Ausubel, que é mais susceptível ao esquecimento. Se o estudante puder ampliar a base de conhecimento potencialmente usável para funcionar como âncora, revendo casos similares existentes em um sistema de Raciocínio Baseado em Caso, ele pode conseguir incorporar tais informações de forma mais significativa, pois terá uma meta ao estudá-las, que é avaliar seu possível uso como elemento num processo de solução de problemas.

Assim, busca-se através deste estudo encontrar as respostas para as seguintes questões:

1. É possível aproveitar a habilidade cognitiva dos alunos talentosos para criar estratégias facilitadoras de ensino e aprendizagem da Matemática e alavancar o desenvolvimento de fluência lógico matemática em estudantes de inteligência normal?

2. Pode um sistema informatizado, tal como o de raciocínio baseado em caso, contribuir para aprimorar o processo de aprendizagem auto-regulatória e aperfeiçoar as habilidades de alunos de inteligência normal ao resolver um problema de matemática?

\section{CONCLUSÃO}

Conclui-se que os estudantes talentosos, deste estudo, destaques ao resolver eficazmente problemas de matemática, possuem algumas habilidades que são requisitos característicos deste grupo de estudantes em Matemática, tais como a habilidade lógicomatemática e intelectual. Ao possuírem esta habilidade, apresentam a capacidade para usar e avaliar relações abstratas, calcular, quantificar, considerar proposições e hipóteses e realizar operações matemáticas complexas, além de sensibilidade a padrões de relacionamento lógicos, funções, afirmações e proposições.

A elicitação dos processos cognitivos dos talentosos na solução de problemas de matemática e o armazenamento desses processos cognitivos numa base de conhecimentos (ou casos) de um Chatterbot, com estratégia de conversação do sistema de raciocínio baseado em caso, que fornece as ferramentas para recuperação e reutilização destas experiências, auxilia os estudantes de inteligência normal durante o processo de aprendizagem da matemática. O pacote de ferramentas do sistema de raciocínio baseado em caso consiste de: (i) repositório para modelagem e armazenamento do banco de casos (estrutura das informações e atributos); (ii) mecanismo de recuperação (ou busca) de casos na base do sistema e, (iii) ferramentas para apresentar a solução dos problemas de matemática.

Os estudantes de inteligência normal, metacognitivamente e heuristicamente, através de um processo de aprendizagem auto-regulada com suporte de andaimes "que guiam os estudantes para fazer predições, experimentar, refletir, escrever explanações, colaborar, contribuir para discussões online, e participar de debates em sala de aula", (Lee et al., 2010), e apoiados pela TIC que possibilita o mecanismo de busca e recuperação de problemas similares, são estimulados para o aprimoramento e conseqüente aquisição das habilidades cognitivas necessárias para resolver um problema de matemática com eficácia.

\section{REFERÊNCIAS}


1. ALVAREZ, Tana Giannasi e PIRES, Inara Martins (2000). Uma nova didática para o ensino de matemática: o método heurístico e a reforma Francisco Campos. Grupo de Trabalho: Educação Matemática, nº 19, PUC São Paulo.

2. AUSUBEL, David P. et al. (1978) Psicologia Educacional, Interamericana Editora, 2 ed.

3. FERNANDES, Teresa Liduina Grigório e VIANA, Tania Vicente Viana (2009) Alunos com necessidades especiais (NEEs): Avaliar para o desenvolvimento pleno de suas capacidades. Est. Aval. Educ., São Paulo, v. 20, n. 43, maio/ago. disponível em: http://www.fcc.org.br/pesquisa/publicacoes/eae/arquivos/1495/1495.pdf

4. GARDNER, Howard (1995). Estruturas da mente: a teoria das inteligências múltiplas. Porto Alegre: ArtMédica.

5. GORODETSKY, M. \& KLAVIR, R. (2003). What can we learn from how gifted/average pupils describe their processes of problem solving? Learning and Instruction,13, p.305-325.

6. JONASSEN, D. H. (2003) Learning to solve problems: an instructional design guide. Pfeiffer.

7. KOLODNER, Janet. (1993) Case-based Reasoning. Morgan Kaufmann Publishers.

8. LEE, H. S. et al., (2010) How do technology-enhanced inquiry science units impact classroom learning? Journal of Research in Science Teaching. vol.47. $\mathrm{n}^{\circ} 1$. pp $71-90$.

9. LEONHARDT, Michelle D. (2005) Doroty: um Chatterbot para treinamento de profissionais atuantes no gerenciamento de redes de computadores. Dissertação de Mestrado. Porto Alegre: UFRGS.

10. LOUREIRO, Cristina et al. (1990). Calculadoras na Educação Matemática: Actividades. Associação de Professores de Matemática. $2^{\mathrm{a}}$ ed.

11. MELCHIORS, Cristina \& TAROUCO, Liane M. R. (1999) Fault management in computer networks using case-based reasoning: DUMBO system. ICCBR-99, LNAI 1650, pp.510-524.

12. POLYA, George (1995) A arte de resolver problemas. $2^{\mathrm{a}}$ ed. Rio de Janeiro: Interciência.

13. ROGERS, K. B. (2007) Lessons Learned About Educating the Gifted and Talented:A Synthesis of the Research on Educational Practice. University of New South Wales: Gifted Child Quarterly, vol. 51, n 4, pp. 382 - 396.

14. STERNBERG, Robert J. \& DAVIDSON, Janet E. (1983) Insight in the gifted. Educational Psychologist, 18 (1), p.51 - 57.

15. SHIH, K.-P., CHANG, C.-Y., CHEN, H.-C., \& WANG,S.-S (2005). A selfregulated learning system with scaffolding support for self-regulated $\mathbf{e} / \mathbf{m}$ learning. Proceedings of the $3^{\text {rd }}$ IEEE International Conference on Information Technology: Research and Education (ITRE 2005), 30 - 34.

16. SHIH, Kuei-Ping, CHEN, Hung-Chang, CHANG, Chih-Yung \& KAO, TainChien (2010). The development and implementation of scaffolding-based self-regulated learning system for e/m-learning. Educational Technology \& Society, 13(1), 80 - 93.

17. TIROSH, D. (2009) What do we know about mathematics teacher education? What evidence do we have? What comes next? Math Teacher Education 12, pp. 83 - 87.

18. VYGOTSKY, L. (2007) A formação social da mente: o desenvolvimento dos processos psicológicos superiores. Martins Fontes: São Paulo. 